\title{
Anatomic variations of the paranasal sinuses in the general pediatric population*
}

Oded Cohen 1,3, Meital Adi2,3, Yael Shapira-Galitz',3, Doron Halperin'1,3, Meir Warman ${ }^{1,3}$

'Department of Otolaryngology, Head and Neck Surgery, Kaplan Medical Center, Rehovot, Israel

2 Department of Radiology, Kaplan Medical Center, Rehovot, Israel

${ }^{3}$ Hebrew University- Hadassah Medical School, Jerusalem, Israel
Rhinology 57: 3, 206 - 212, 2019

https://doi.org/10.4193/Rhin18.193

*Received for publication:

August 23, 2018

Accepted: September 21, 2018

\begin{abstract}
Background: The prevalence of sinuses' anatomic variations in the healthy pediatric population has not been studied. The study describes the prevalence of known anatomic variations with regard to gender and age in this population.
\end{abstract}

Methods: A single academic institute observational cohort study. A total of 200 head CT scans were reviewed, subdivided into five equal age subgroups (0-4.99; 5-7.99; 8-10.99; 11-13.99; $14-17$ years), with an equal male to female ratio. Different subgroups were randomly assigned to two senior residents (100 CTs each). A senior rhinologist and radiologist were randomly selected to review 100 CTs each. Consensus was reached after a joint review. Each CT was evaluated for the presence of sinuses and the following variations: deviated septum, frontoethmoidal, infraorbital, posterior-ethmoid cells (Kuhn, Haller, and Onodi cells, respectively) and concha bullosa. Definitions were made according to the European Position on Rhinosinusitis 2012.

Results: Gender did not affect sinus development or anatomical variations. The frontal and sphenoid sinuses were significantly less developed in the 0-4.99 years group. The point prevalence of concha bullosa and deviated septum significantly increased with age. The point prevalence of Haller cells demonstrated borderline significance among age groups, with children 0-4.99 demonstrating the lowest point prevalence. A significant association was found between the existence of Haller cells to Kuhn and Onodi cells.

Conclusions: Anatomical variations should be expected in the pediatric population. Familiarity with their point prevalence and associations may assist pediatric endoscopic sinus surgery planning.

Key words: concha bullosa, Haller, Kuhn, Onodi, pediatric sinus, variation

\section{Introduction}

The anatomical understanding of the paranasal sinuses has changed dramatically since the introduction of computerized tomography (CT). CTs have allowed physicians to appreciate paranasal sinus development, the extent of pneumatization as well as visualization of posterior sinuses, primary obstruction sites and anatomical variants ${ }^{(1)}$.

Anatomical variants are common, yet there is a debate whether their existence results in rhinosinusitis ${ }^{(2)}$. Regardless of their controversial role in the pathogenesis of rhinosinusitis, anatomic variants are important for planning functional endoscopic sinus surgery (FESS). The proximity of certain 'atypical' ethmoidal cells such as infraorbital (Haller), Sphenoethmoidal (Onodi) and Frontoethmoidal (Kuhn) cells to the orbit, optic nerve and base of skull (respectively) mandate an absolute familiarity of the surgeon with these structures, their incidence and point prevalence in different age groups.

Several studies investigated the point prevalence of anatomic variations in children with chronic sinusitis ${ }^{(3,4)}$. To the best of our knowledge, no study has focused on general pediatric population. Moreover, the majority of these studies were age unbalanced $^{(3-6)}$ and $\backslash$ or gender unbalanced ${ }^{(3-5)}$. As a result, the data of such variations in the general pediatric population is not well established. 
Anatomic variants are important for FESS planning ${ }^{(1,2)}$. FESS for the pediatric population has been shown to be a safe and effective procedure for chronic sinusitis ${ }^{(6)}$ as well as a neurosurgical approach to the skull base ${ }^{(7)}$. The population for the latter indication is expected to have normal sinus anatomy and variations, yet this is not well established in the literature to the best of our knowledge.

The aim of this current study was to determine the rate of sinonasal anatomical variants in a general pediatric population. It is a large scale, CT based anatomical study designed to be gender and age balanced.

\section{Patients and Methods}

This is a retrospective, case series study of pediatric patients who underwent a head CT scan at our institute's emergency department (ED) between January and May 2017. Indications for CT scans were trauma and severe headache justifying a CT scan by senior ED pediatrician. The study was approved by our Institutional Ethics Committee and the study design is presented in Figure 1. CT scans with radiologic evidence of previous interventions (e.g. FESS), CT scans with opacification precluding assessment of the anatomic variations detailed below, $\mathrm{CT}$ scans of poor technical quality or CTs which were indicated for trauma and with evidence of injury to the sinu-nasal system (e.g. nasal fracture, hemosinus suspicion etc.) were excluded. CTs were chosen on a consecutive basis if it fulfilled the inclusion criteria and was based on the following division: Age was categorized into five age groups, each of 40 CT scans (overall, 200 CT scans), based on Wolf et al. ${ }^{(8)}$ with a few modifications: (1) 0-4.99 years; (2) 5-7.99; (3) 8-10.99; (4) 11-13.99 and (5) 14-17 years. All age subgroups had 1:1 male to female ratio. The subgroups were

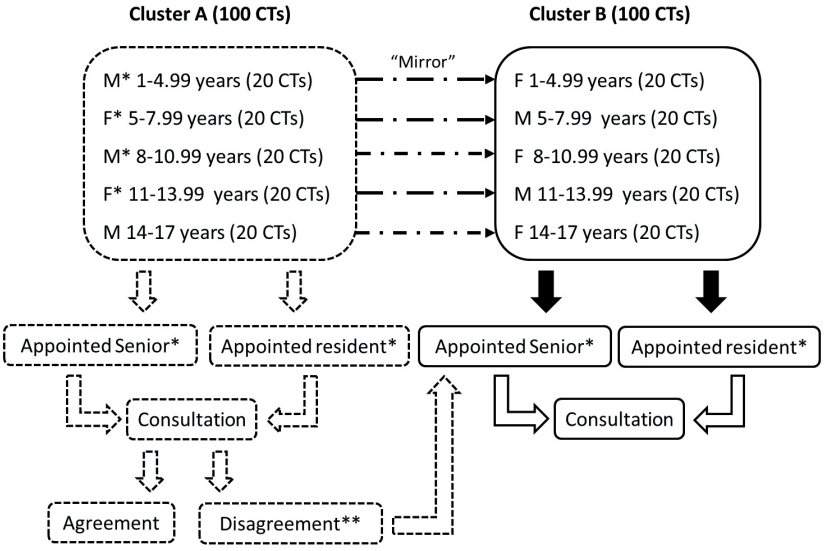

Figure 1. Study Design. Each cluster was reviewed by both resident and senior. If disagreement appeared, a consultation with the other senior was made. M - Male; F - Female; * - randomization; **- applied for cluster $\mathrm{B}$ as well.

randomly and equally divided between two senior residents (OC and YSG), with each resident analyzing a total of 100 CTs (a cluster). Division into age subgroups of the 100 CTs was semi-randomized in the first cluster (Figure 1). The other cluster consisted of a 'mirror image' of the initial cluster (for example, if one cluster contained males 8-11 years old, then the other cluster contained CTs of females 8-11 years old). In the end, each cluster contained all age subgroups with a random distribution of gender (3:2 in each cluster). The coupling of a resident to a cluster was made randomly (by flipping a coin). Each resident was teamed with a specialist, either a senior rhinologist (MW) or a senior radiologist (MA) randomly selected as well. Both the resident and specialist scored the scans. Consensus was achieved after joint review by

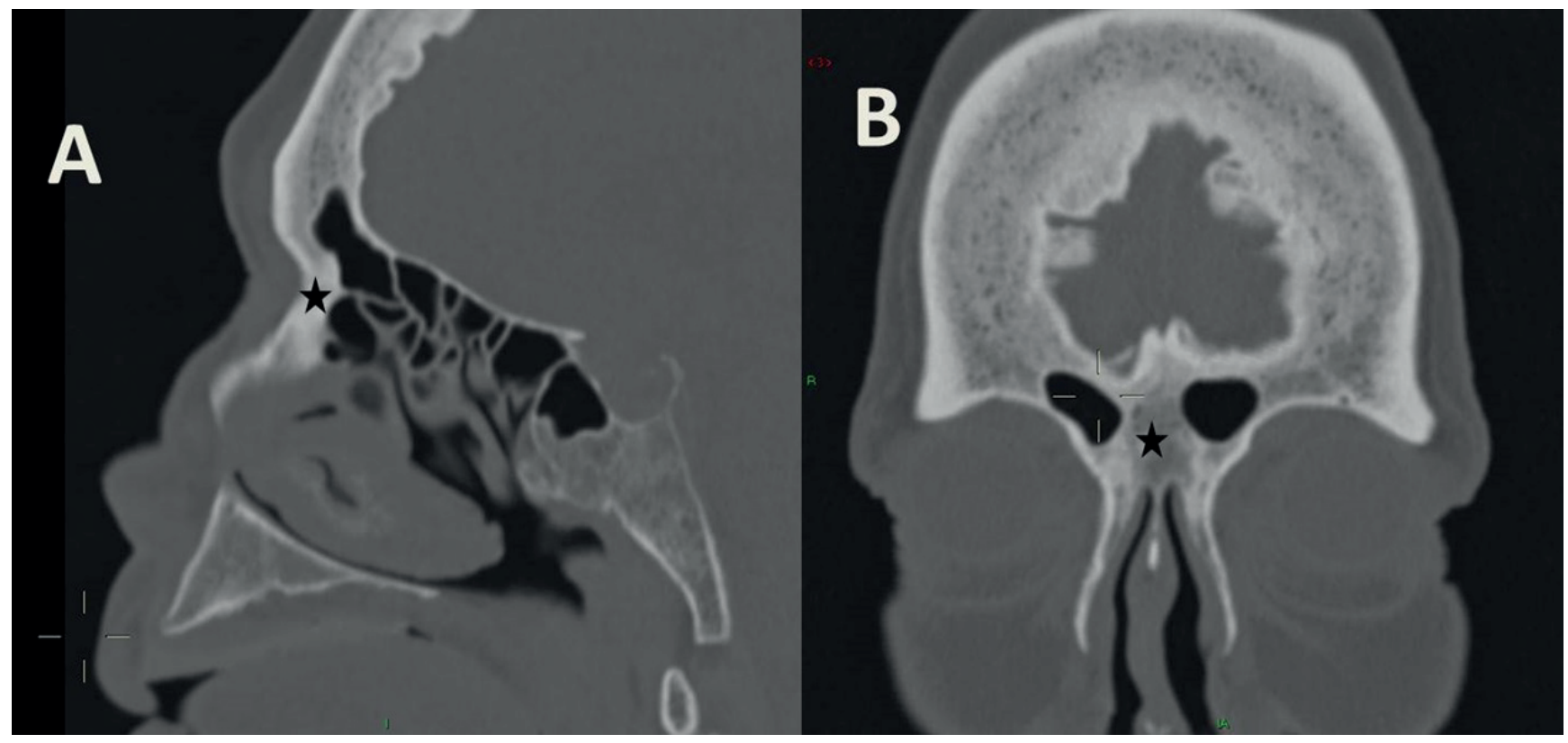

Figure 2. A + B: Frontal Sinus. Frontal sinus pneumatized above the frontal beak (black star) in a 2 years old girl. A - Sagittal view; B - Coronal view. 


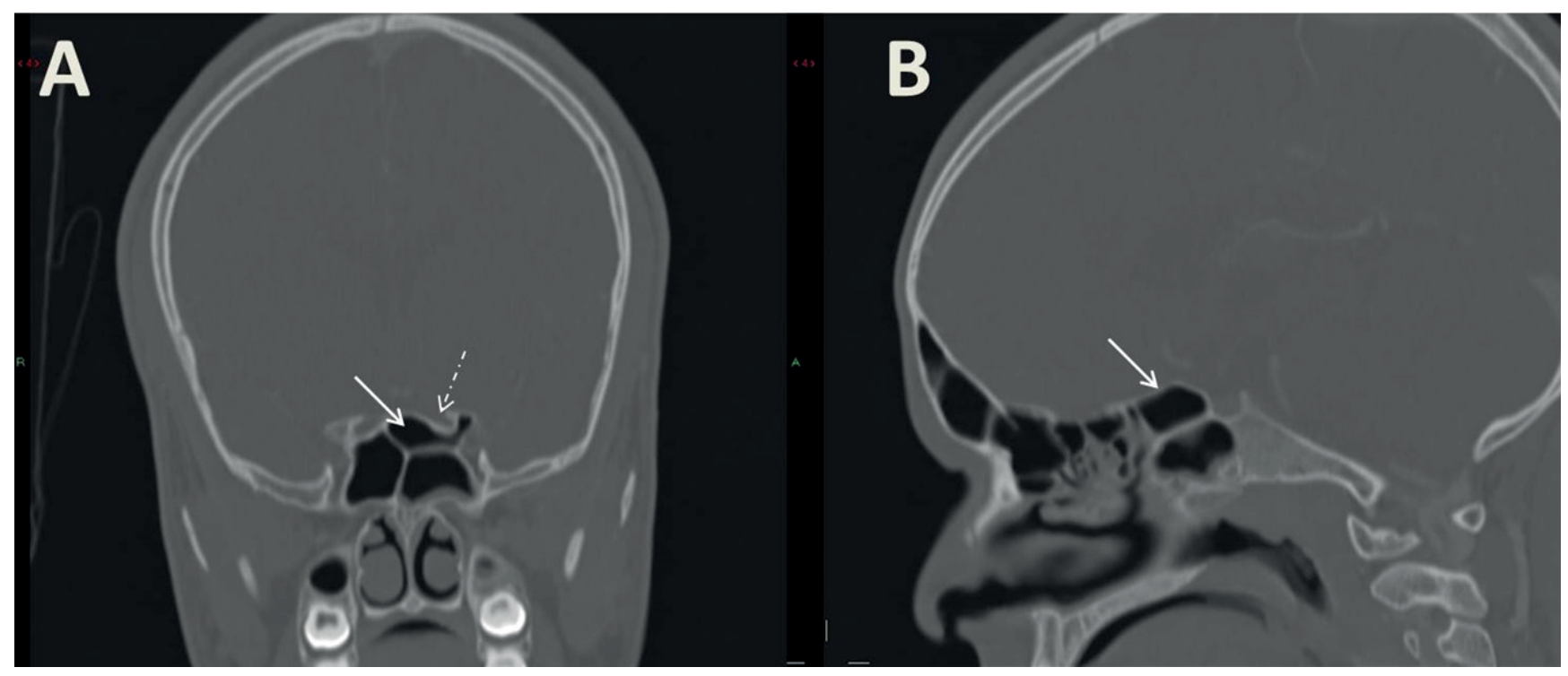

Figure 3. A + B: Posterior Ethmoid Cell (Onodi) cell. Posterior Ethmoid Cell (Onodi) cell (white arrow) pneumatized above the sphenoid sinus in a 15 years old girl. The optic nerve is marked by a dotted arrow. A - Coronal view; B - Sagittal view.
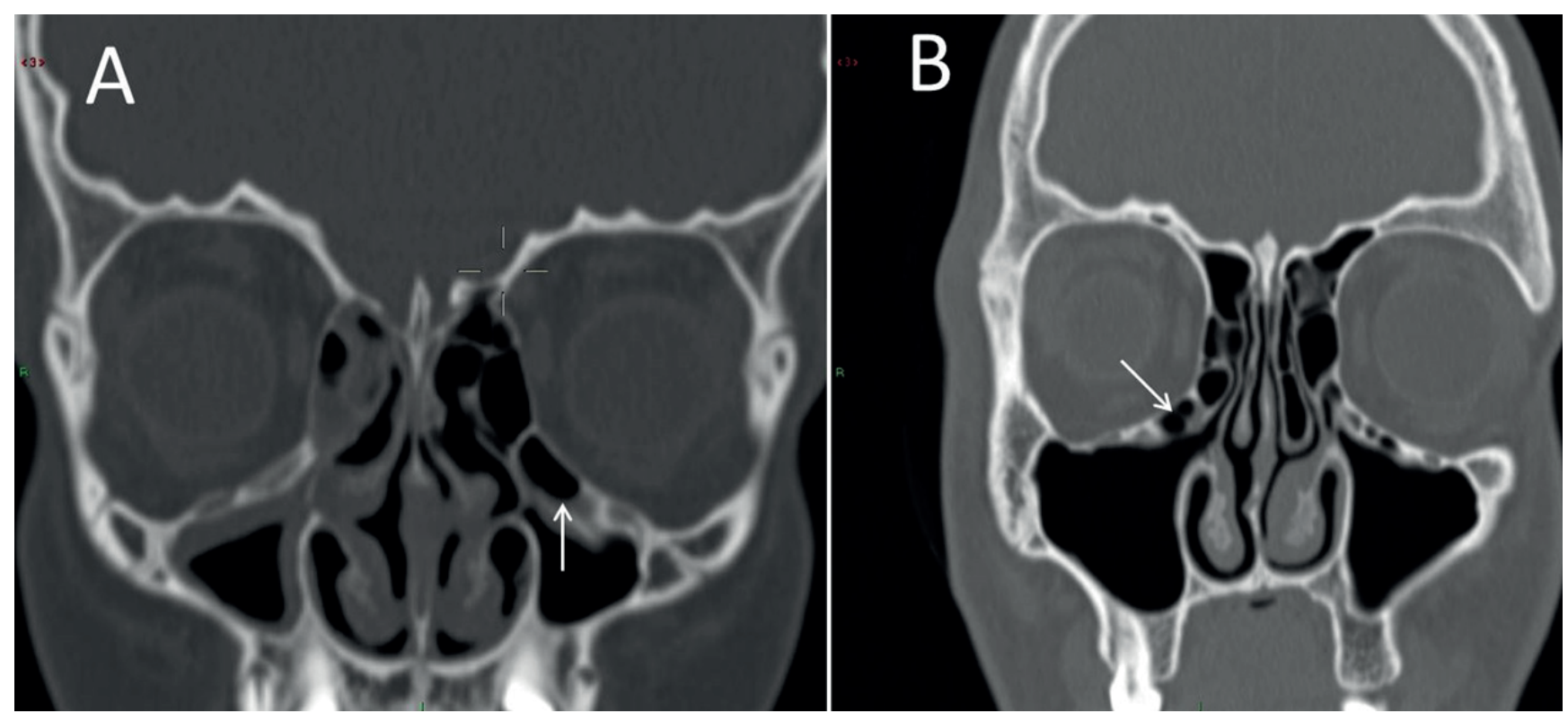

Figure 4. A + B: Infraorbital (Haller) cell. Infraorbital (Haller) cell in white arrow. A - 10 years old boy; B - 15 years old girl.

both. In cases where the resident and the senior physician could not reach an agreement after a joint review, a joint consultation with the other senior was conducted and a decision was made based on the majority decision.

All CT scans were carried out using the Philips Brilliance IDT 64 slice or iCT 256 slice scanner (Brilliance 64, Philips Healthcare, Cleveland, $\mathrm{OH}, \mathrm{USA}$ ) and stored using a picture archiving and communication system (Carestream PACS, Carestream Health, Inc. Rochester, NY, USA). Helical scans were acquired at 0.625 $\mathrm{mm}$ slice collimations, $2 \mathrm{~mm}$ slice thickness, $1 \mathrm{~mm}$ increments ( 3 $\mathrm{mm}$ and $1.5 \mathrm{~m}$, respectively, for physically larger children), 120 $\mathrm{kVp}$ and tube current of 50-270 mAS (0-3 years about $88 \mathrm{mAS}$;
3-12 about 128 mAS and 269 mAS for older children).

Each CT was evaluated for the existence of each sinus (right and left frontal, ethmoid, maxillary and sphenoid) and the following variations: deviated nasal septum, concha bullosa, Kuhn (frontoethmoidal), Haller (infraorbital) and Onodi (sphenoethmoidal) cells. Anatomical definitions were made based on the European Position Paper on the Anatomical Terminology of the Internal Nose and Paranasal Sinuses ${ }^{(2)}$. Existence of a sphenoid sinus was defined if aeriation posterior to the rostrum of the sphenoid bone was detected. Existence of a frontal sinus was defined if aeriation superior to a noticeable frontal beak was detected (Figure 2A and 2B). Concha bullosa was defined as aeration 
Table 1. Gender differences in prevalence of sinuses and anatomical variants.

\begin{tabular}{|c|c|c|c|c|}
\hline $\begin{array}{l}\text { Sinus I Anatomi- } \\
\text { cal Variant }\end{array}$ & & $\begin{array}{c}\text { Male } \\
(n=100)\end{array}$ & $\begin{array}{l}\text { Female } \\
(n=100)\end{array}$ & $\begin{array}{c}P \\
\text { value }\end{array}$ \\
\hline \multirow{2}{*}{ Frontal } & Unilateral & 9 & 15 & \multirow{2}{*}{ NS } \\
\hline & Bilateral & 63 & 59 & \\
\hline \multirow{2}{*}{ Ethmoid } & Unilateral & 0 & 0 & \multirow{2}{*}{ NS } \\
\hline & Bilateral & 100 & 100 & \\
\hline \multirow{2}{*}{ Maxillary } & Unilateral & 0 & 0 & \multirow{2}{*}{ NS } \\
\hline & Bilateral & 98 & 100 & \\
\hline \multirow{2}{*}{ Sphenoid } & Unilateral & 8 & 2 & \multirow{2}{*}{ NS } \\
\hline & Bilateral & 75 & 80 & \\
\hline \multirow{2}{*}{ Concha Bullosa } & Unilateral & 21 & 20 & \multirow{2}{*}{ NS } \\
\hline & Bilateral & 9 & 5 & \\
\hline \multirow{2}{*}{ Khun cells* } & Unilateral & $18(25 \%)$ & $15(20.3 \%)$ & \multirow{2}{*}{ NS } \\
\hline & Bilateral & $14(19.4 \%)$ & $8(10.8 \%)$ & \\
\hline \multirow{2}{*}{ Haller cells } & Unilateral & 12 & 14 & \multirow{2}{*}{ NS } \\
\hline & Bilateral & 12 & 10 & \\
\hline \multirow{2}{*}{ Onodi cells ${ }^{* *}$} & Unilateral & $12(14.5 \%)$ & $4(4.9 \%)$ & \multirow{2}{*}{ NS } \\
\hline & Bilateral & $4(4.8 \%)$ & $6(7.3 \%)$ & \\
\hline Deviated Septum & & 39 & 30 & NS \\
\hline
\end{tabular}

If not specified, values represent both numbers and percentage. Khun and Onodi cells were adjusted to the existence of frontal and sphenoid sinuses, respectively. ${ }^{*} n=146 ;{ }^{* *} n=165$.

within the vertical part of the middle turbinate. An Onodi cell was defined as a posterior ethmoidal cell which develops lateral and/or superiorly to the sphenoid sinus (Figure $3 \mathrm{~A}$ and $3 \mathrm{~B}$ ). A Haller cell was defined as any ethmoidal cell which pneumatizes inferior to the orbital floor and lateral to a line parallel with the lamina papyracea (Figure 4A and 4B). Kuhn cells were defined as an ethmoidal cell superior to the Agar Nasi cell. For the purpose of simplicity, and considering the lack of consensus ${ }^{(2)}$, classification of the specific subtype of Kuhn \frontoethmoidal cells was avoided ${ }^{(2,9)}$ and existence of any type was considered as positive. Point prevalence of Kuhn and Ondoi cells were adjusted to the development of a frontal and sphenoid sinus, respectively. Similar to previous studies ${ }^{(10)}$, definitions of a significant deviated septum was a matter of judgment. However, any deviated septum resulting in contact between the septum and concha media/ lateral wall or narrowing of the medial corridor was considered as positive. All CTs were observed in axial, coronal and sagittal views before scoring.

\section{Statistical analyses}

Statistical analyses were performed with Statistical Package for the Social Sciences package version 21.0 (SPSS Inc., Chicago, IL, USA). The numeric variables were presented as total numbers, percentages, and mean \pm standard deviation (SD) values. Normally distributed values are represented by mean and SD. A paired $T$ test was used to compare non-categorical continuous variables in both sides of the same sinus. Categorical variables were compared using a Fisher exact test or Chi-square test, as appropriate. A two-tailed $\mathrm{P}$ value $<0.05$ was considered significant.

\section{Results}

A total of 200 CTs were scanned, divided to five equal age subgroups as detailed in the Methods section. No significant differences were found in sinus development or anatomic variation with regards to gender (Table 1).

The development of the sinuses according to age is presented in Table 2. Except for two cases of undeveloped maxillary sinuses, all maxillary and ethmoid sinuses were found from the youngest age group and up. The sphenoid sinus showed increased development starting from age 5. Children younger than five years old had a significantly lower point prevalence of sphenoid sinus compared to all other age groups ( $27.5 \%$ vs. $90 \%$ [5-7.99 years] ; 97.5\% [8-13.99] ; 100\% [14-17] ; $p$-value <0.001 for each age group, chi square test). No differences were found when comparing the second age group (5-7.99) to the children aged 8-13.99. The oldest children (14-17) demonstrated significantly higher point prevalence of the sphenoid sinus when compared to children aged $5-8(100 \%$ vs. $90 \% p=0.04)$. Similar to the sphenoid sinus, the frontal sinus demonstrated increased development starting from age five, though development was more gradual during ages 5-8 years. This was demonstrated by a significant difference between the smallest age subgroup to all other subgroups (15\% vs. $72.5 \%$ [5-7.99 years]; 92.5\% [8-10.99]; 95\% [11-13.99; 90\% [14-17]; p-value <0.001 for each age group, chi square test). However, children aged 5-8 also demonstrated a significant difference to all other aged group ( $p$-value $0.03,0.006$ and 0.045 , respectively).

Anatomic variations with regard to lateralization and age are presented in Table 2. Concha bullosa was found in 55 cases (27.5\%), of which 14 (7\%) were bilateral. Kuhn cells were found in 58 cases (37.7\%), of which 22 (15.1\%) were bilateral. Haller cells were found in 48 cases (24\%), of which $22(11 \%)$ were bilateral. Onodi cells were found in 26 cases (15.7\%), of which 10 (6\%) were bilateral. Gender did not affect sinus variation (Table 1). The distribution of anatomic variations according to age groups is detailed in Table 2. No significant differences in the presence of Kuhn and Onodi cells were found between the different age subgroups. A borderline significance ( $p$ value $=0.054$ ) was found in the point prevalence of Haller cells between different age groups. The youngest age group (0-4.99 years) had a significantly lower rate of Haller cells compared to 
Table 2. Age differences in prevalence of sinuses and anatomical variants.

\begin{tabular}{|c|c|c|c|c|c|c|c|c|}
\hline \multicolumn{2}{|c|}{ Sinus I Anatomical Variant } & \multicolumn{6}{|c|}{ Age, years; n (\%) } & \multirow[t]{2}{*}{ P value } \\
\hline & & Total & $0-4.99$ & $5-7.99$ & 8-10.99 & 11-13.99 & 14-17 & \\
\hline \multirow{2}{*}{ Frontal } & Unilateral & $24(12)$ & $2(5)$ & $7(17.5)$ & $5(12.5)$ & $6(15)$ & $4(10)$ & \multirow{2}{*}{$<0.001$} \\
\hline & Bilateral & $122(61)$ & $4(10)$ & $22(55)$ & $32(80)$ & $32(80)$ & $32(80)$ & \\
\hline \multirow{2}{*}{ Ethmoid } & Unilateral & 0 & $0(0)$ & $1(0)$ & $2(0)$ & $3(0)$ & $4(0)$ & \multirow{2}{*}{ NS } \\
\hline & Bilateral & $200(100)$ & $40(100)$ & $41(100)$ & 42 (100) & $43(100)$ & 44 (100) & \\
\hline \multirow{2}{*}{ Maxillary } & Unilateral & $0(0)$ & $0(0)$ & $0(0)$ & $0(0)$ & $0(0)$ & $0(0)$ & \multirow{2}{*}{ NS } \\
\hline & Bilateral & $198(99)$ & $39(97.5)$ & $40(97.5)$ & $40(100)$ & $41(100)$ & $42(100)$ & \\
\hline \multirow{2}{*}{ Sphenoid } & Unilateral & $10(5)$ & $2(5)$ & $3(7.5)$ & $0(0)$ & $3(7.5)$ & $2(5)$ & \multirow{2}{*}{$<0.001$} \\
\hline & Bilateral & $155(77.5)$ & $9(22.5)$ & $33(82.5)$ & 39 (97.5) & $36(90)$ & $38(95)$ & \\
\hline \multirow{2}{*}{$\begin{array}{l}\text { Concha Bul- } \\
\text { losa }\end{array}$} & Unilateral & $41(20.5)$ & $4(10)$ & $9(22.5)$ & $6(15)$ & $11(27.5)$ & $11(27.5)$ & \multirow{2}{*}{0.029} \\
\hline & Bilateral & $14(7)$ & $1(2.5)$ & $2(5)$ & $2(5)$ & $4(10)$ & $5(12.5)$ & \\
\hline \multirow{2}{*}{ Khun cells* } & Unilateral & $33(22.6)$ & $2(33.3)$ & $4(13.8)$ & $10(27)$ & $10(26.3)$ & $7(19.4)$ & \multirow{2}{*}{ NS } \\
\hline & Bilateral & $22(15.1)$ & $0(0)$ & $4(13.8)$ & $3(8.1)$ & $7(18.4)$ & $8(22.2)$ & \\
\hline \multirow{2}{*}{ Haller cells } & Unilateral & $26(13)$ & $2(5)$ & $8(20)$ & $3(7.5)$ & $9(22.5)$ & $4(10)$ & \multirow{2}{*}{0.054} \\
\hline & Bilateral & $22(11)$ & $2(5)$ & $2(5)$ & $6(15)$ & $4(10)$ & $8(20)$ & \\
\hline \multirow{2}{*}{ Onodi cells ${ }^{* *}$} & Unilateral & $16(9.7)$ & $0(0)$ & $4(11.1)$ & $3(7.7)$ & $3(7.7)$ & $6(15)$ & \multirow{2}{*}{ NS } \\
\hline & Bilateral & $10(6)$ & $1(9.1)$ & $00)$ & $4(10.3)$ & $2(5.1)$ & $3(7.5)$ & \\
\hline \multicolumn{2}{|c|}{ Deviated Septum } & $69(34.5)$ & $5(12.5)$ & $9(22.5)$ & $17(42.5)$ & $21(52.5)$ & 17 (42.5) & 0.01 \\
\hline
\end{tabular}

Khun and Onodi cells were adjusted to the existence of frontal and sphenoid sinuses, respectively. ${ }^{*} \mathrm{n}=146 ;{ }^{* *} \mathrm{n}=165$

children aged 11-13.99 years ( $p$-value=0.041) and a borderline significant difference was found when compared to the oldest age group (14-17 years; $p$-value $=0.072$ ). Point prevalence of concha bullosa significantly differed between age groups ( $p$ value $=0.029$ ). The youngest age group had significantly lower rates of concha bullosa compared with children 11-13.99 (12.5\% vs. $37.5 \%, p$-value $=0.01)$ and children above 14 years $(12.5 \%$ vs. $40 \%$, $p$-value $=0.005$ ). No difference was found between children aged 5-7.99 and the other older age groups. Septal deviation was also found to significantly increase after the age of 8 years ( $p$-value $<0.001)$. The point prevalence of a deviated septum was significantly lower in the youngest age group (1-4.99) compared with ages 8 and above ( $p$-value $=0.003,<0.001,<0.001$ respectively). No significant difference was found between children aged 1-4.99 and 5-7.99 years. Children aged 5-7.99 years had significantly lower point prevalence of a deviated septum compared to children 11-13.99 (22.5\% vs.52.5\%, p-value=0.006), and a borderline significance when compared to the other older aged groups ( $22.5 \%$ vs. $42.5 \%$ [both groups], p-value $=0.056)$. Finally, we looked for significant associations between different anatomic variations. A significant association was found between Haller and Kuhn cells ( $p$-value<0.001; OR 5.7, Cl 2.612.4) and between Haller and Onodi cells ( $p$-value $=0.005$; OR 3.3, Cl 1.4-7.7).

\section{Discussion}

The aim of this study was to determine the rate of sinonasal anatomical variants in a normal pediatric population. Existence of anatomical variations should be considered when suggesting a sinus surgery for a child, and off course are critical in the pre-operative planning for FESS surgery. By selecting a general population and not children with chronic rhinosinusitis, relying on advanced CT technologies and by designing large and balanced subgroups, the study expands our knowledge of the point prevalence of anatomic variants among the pediatric population. Our study's results show that anatomic variants occur from an early age, yet the point prevalence of Kuhn and Onondi cells do not seem to differ significantly between the age subgroups. We found that concha bullosa and septal deviation point prevalence significantly increases with age, as well as Haller cells ( $p$-value=0.054). Moreover, we found an association between Haller, Kuhn and Onodi cells. This association may be important for planning nasal endoscopic surgeries for both children with chronic rhinosinusitis and those requiring an endoscopic approach to the skull base.

It seems that an increased point prevalence of deviated nasal septum takes place around the age of 8 years old. Reported point prevalence of deviated nasal septum in childhood and adulthood range greatly from about $10 \%$ and up to $90 \%{ }^{(1)}$. A large study of 4,090 neonates on neonatal nasal septum 
deformity has shown a point prevalence of $0.93 \%$, which led the authors to question the role of birth as a possible etiology ${ }^{(12)}$. In older children, a point prevalence of $12.4 \%$ was noted in children aged $2.5-6$ years ${ }^{(13)}$, similar to the rate found in our youngest age group. Reitzen et al. also found that nasal deformity occurs in a higher frequency in older children and adults ${ }^{(14)}$. To the best of knowledge, no study has yet to describe the age from which deviated septum point prevalence increases rapidly. Altogether, it seems that deviated nasal septum is probably acquired in late childhood and adolescence rather than intrauterine or from early childhood trauma.

Concha bullosa also significantly increased with age, though it seems pneumatization of the concha media starts after the age of five, and its point prevalence generally increases throughout the years. In the older age groups, the rate of concha bullosa was $37.5-40 \%$. These rates are similar to those reported by Kim et al. (39.4\%; $n=94$; mean age $11.2 \pm 2.6$ years) ${ }^{(3)}$, but significantly higher than the $10-24 \%$ reported in other studies $(5,10,15,16)$. This rate is comparable with reported rates for adults whose range is $35-44 \%{ }^{(1,17)}$. Based on our study design, it seems that natural course of concha bullosa is that it develops in early childhood and stabilizes in adolescence. Similar to our finding, Unlu et al. found that concha bullosa becomes more apparent after the age of 7-8 years ${ }^{(18)}$. The exact reason of pneumatization of the middle turbinate is unknown yet. The ethmoids are considered to be responsible for pneumatization of concha bullosa ${ }^{(18,19)}$, yet this does not explain the difference in which pneumatization is noted in the ethmoids (at birth) vs. concha bullosa (early childhood). We hypothesize that the growth of the skull, and nasal cavity in particular, may allow for the expansion of the middle concha by pneumatization. Further studies are required to expand our understanding of concha bullosa and deviated septum development. Our study did not find an association between nasal septum deformity and concha bullosa, in contrast to previous studies held in the adult population ${ }^{(17)}$. Whether this observation represents a true difference between adult and pediatric populations should be a subject for future anatomical studies.

A novel contribution of this current study is the association found between some atypical ethmoid cells. Such associations were found between Haller and Kun cells and Haller and Onodi cells. A literature search did not come up with another study of pediatric paranasal sinus anatomy with similar results. These associations are a novel finding, and to the best of our knowledge have not been previously reported. This information is of clinical importance for pediatric FESS surgeons. Knowledge of such potential associations should improve surgical planning and may help in avoiding unplanned complications.

A second aim of this study was to evaluate the development of the pediatric paranasal sinuses, and this topic has been much better studied throughout the years ${ }^{(8,20)}$. Our results reaffirmed previously reported studies ${ }^{8,20,21)}$. We have found that the sphenoid and the frontal sinuses develop later than the maxillary and ethmoids, with significant differences found in children under five years old. Conversely, Adibelli et al. conducted an MRI imaging analysis of sinus development and found the frontal sinus pneumatization starts at five years but the sphenoid sinus at nine months ${ }^{(22)}$. In agreement with previous studies, gender did not seem to affect sinus development per se, yet volume differences were noted both in pediatric ${ }^{(21,22)}$ and adult patients (23,24).

The study's strengths are its large cohort, its balanced subgroups with regard to age and gender, its multiple slides and views (1-2mm thickness; all coronal, axial and sagittal views were reviewed) and its blinded double review of each cluster (a 100 CTs, balanced by age and gender). Nevertheless, some limitations still exist. Not all CTs were reviewed by all authors, with each cluster being reviewed by a resident (senior resident) and a single senior. Important abnormalities such as paradoxical middle turbinate, depth of lamina cribrosa (Keros classification) were not assessed. The study addresses the presence/absence of the sinuses was evaluated, without referring to the dimension/ structure of a sinus, which could have contributed additional insights to the development of the paranasal sinuses. The CT scans were performed during winter season, thus increasing possibility of an acute sinusitis. Symptoms and clinical condition at the time of CT scan were not evaluated. This in turn may have exposed the data for potential biases. However, when considering that the aim of the study was to describe the point prevalence of anatomic variations regardless of clinical impact and the study's large cohort, we feel such exposure does not impair the validity of the study's results. Future, matched, case-control studies should compare point prevalence of anatomical variants between pediatric with vs. without signs and $\backslash$ or symptoms of sinusitis. Finally, the study did not refer to the ethnic variation of the population, which has been shown to impact the anatomy of paranasal sinus and its variations ${ }^{(25,26)}$, yet is should be related to the Caucasian population, which represents more than $95 \%$ of our municipal population ${ }^{(27)}$.

\section{Conclusions}

Sinonasal anatomical variations should be expected in the pediatric population. Familiarity with their point prevalence and associations between different variations may contribute towards better pediatric endoscopic surgery planning.

\section{Authorship contribution}

OC: Substantial contributions to the conception and design of the work; acquisition, analysis, and interpretation of data for the work; drafting the work; final approval of the version to be published; agreement to be accountable for all aspects of the work in ensuring that questions related to the accuracy and 
integrity of any part of the work ar:e appropriately investigated and resolved; MA: Substantial contributions to the conception ; design of the work ; the acquisition of data for the work; revising it critically for important intellectual content; final approval of the version to be published; agreement to be accountable for all aspects of the work in ensuring that questions related to the accuracy of any part of the work are appropriately investigated and resolved. YSG: Substantial contributions to the conception ; design of the work and the acquisition of data for the work; revising it critically for important intellectual content; final approval of the version to be published; agreement to be accountable for all aspects of the work in ensuring that questions related to the integrity of any part of the work are appropriately investigated and resolved. DH: Interpretation of data for the work; revising it critically for important intellectual content; final approval of the version to be published; agreement to be accountable for all aspects of the work in ensuring that questions related to the accuracy and integrity of any part of the work are appropriately investigated and resolved. MW: Substantial contributions to the conception and design of the work and the acquisition of data for the work; revising it critically for important intellectual content; final approval of the version to be published; agreement to be accountable for all aspects of the work in ensuring that questions related to the accuracy and integrity of any part of the work are appropriately investigated and resolved.

\section{Conflict of interest}

The authors declare no conflict of interests exist regarding the study.

\section{References}

1. Nouraei SA, Elisay AR, Dimarco A, et al. Variations in paranasal sinus anatomy implications for the pathophysiology of chronic rhinosinusitis and safety of endoscopic sinus surgery. J Otolaryngol Head Neck Surg. 2009;38(1):32-37.

2. Lund VJ, Stammberger $H$, Fokkens WJ et al. European position paper on the anatomical terminology of the internal nose and paranasal sinuses-a free resource. Rhinol Suppl. 2014;24:1-34.

3. Jun Kim H, Jung Cho M, Lee J-W, et al The relationship between anatomic variations of paranasal sinuses and chronic sinusitis in children. Acta Otolaryngol. 2006;126(10):1067-1072.

4. Van Der Veken PJV, Clement PAR, Buisseret $T$, Desprechins B, Kaufman L, Derde MP CT-scan study of the incidence of sinus involvement and nasal anatomic variations in 196 children. Rhinology. 1990;28(3):177184.

5. Lusk RP, McAlister B FA. Anatomic variation in pediatric chronic sinusitis. Otolaryngol Clin North Am. 1996;29(1):75-79.

6. April MM1, Zinreich SJ, Baroody FM NR. Coronal CT scan abnormalities in children with chronic sinusitis. Laryngoscope. 1993;103(9):985-990.

7. Hebert RL, Bent JP. Meta-analysis of out comes of pediatric functional endoscopic sinus surgery. Laryngoscope. 1998;108(6):796-799.

8. Rastatter JC, Snyderman CH, Gardner PA et al. Endoscopic endonasal surgery for sinonasal and skull base lesions in the pediatric population. Otolaryngol Clin North Am. 2015;48(1):79-99.

9. Wolf G, Anderhuber W, Kuhn F. Development of the paranasal sinuses in children: implications for paranasa sinus surgery. Ann Otol Rhinol Laryngol. 1993;102(9):705-711.
10. Bent JP, Guilty-Siller G, Kuhn FA. The Frontal Cell As a Cause of Frontal Sinus Obstruction. Am J Rhinol. 1994;8(4):185-191.

11. Mladina R, Cujić E, Subarić M et al. Nasal septal deformities in ear, nose, and throat patients: an international study. Am J Otolaryngol. 2008;29(2):75-82

12. Podoshin L, Gertner $R$, Fradis $M$ BA. Incidence and treatment of deviation of nasal septum in newborns. Ear Nose Throat J. 1991;70(8):485-487.

13. Van Cauwenberge P DA. The relationship between nasal and middle ear pathology. Acta Otorhinolaryngol Belg. 1983;37(6):830841

14. Reitzen SD, Chung W, Shah AR. Nasal septal deviation in the pediatric and adult populations. Ear Nose Throat J. 2011:90(3):112-115.

15. Milczuk HA, Dalley RW, Wessbacher FW RM Nasal and paranasal sinus anomalies in children with chronic sinusitis. Laryngoscope. 1993;103(3):247-252.

16. Willner A, Choi SS, Vezina LG et al. Intranasal anatomic variations in pediatric sinusitis. Am J Rhinol. 1997;11(5):355-360.

17. Stallman JS, Lobo JN, Som PM. The incidence of concha bullosa and its relationship to nasal septal deviation and paranasal sinus disease. Am J Neuroradiol. 2004;25(9):1613-1618.

18. Unlü HH, Akyar S, Caylan R NY. Concha bullosa. J Otolaryngol. 1994;23(1):23-27.

19. Bolger WE, Butzin CA, Parsons DS. Paranasal Sinus Bony Anatomic Variations and Mucosal Abnormalities. Laryngoscope. 1991;101(1):56-64

20. Park IH, Song JS, Choi $H$, et al. Volumetric study in the development of paranasal sinuses by CT imaging in Asian: A Pilot study. Int J Pediatr Otorhinolaryngol. 2010;74(12):1347-1350.

21. Spaeth J, Krügelstein U, Schlöndorff G. The paranasal sinuses in CT-imaging: Development from birth to age 25 . Int $J$
Pediatr Otorhinolaryngol. 1997;39(1):25-40.

22. Adibelli ZH, Songu M, Adibelli H. Paranasal sinus development in children: A magnetic resonance imaging analysis. Am J Rhinol Allergy. 2011;25(1):30-35

23. Cohen O, Warman $M$, Fried $M$, et al. Volumetric analysis of the maxillary, sphenoid and frontal sinuses: A comparative computerized tomography based study. Auris Nasus Larynx. 2018;45(1):170-174.

24. Kawarai, Kkunihiro Fukushima, Teruh Y. Volume Quantification of Healthy Paranasal Cavity by Three-Dimensional CT Imaging. Acta Otolaryngol. 1999;119(540):45-49.

25. Lien C, Weng $H$, Chang $Y$, Lin $Y$. Computed Tomographic Analysis of Frontal Recess Anatomy and its Effect on the Development of Frontal Sinusitis. Laryngoscope. 2010;120(December 2010):2521-2527.

26. Badia L, Lund VJ, Wei W, Ho WK. Ethnic variation in sinonasal anatomy on CT-scanning. Rhinology. 2005:43(October 2005):210-214.

27. Israel Central Bureau of Statistics, The Ethiopian Population in Israel, November 2016.

\section{Oded Cohen}

Address: Kaplan Medical Center

$\mathrm{POB}$

1 Rehovot 76100

Israel

Mobile phone: +972-54-4682490

Telephone no.: +972-8-9441649

Fax no.: +972-8-9441794

E-mail: oded915@gmail.com 